\title{
Does Chronic Cola Consumption Increase Urinary Stone Risk? Evidence from the Drosophila Model of Urolithiasis
}

\author{
Kao-Sung Tsai ${ }^{1,2,3}$, Yung-Hsiang Chen ${ }^{1,2,4}$, Jui-Lung Shen ${ }^{5,6}$, Kee-Ming Man ${ }^{7,8}$, Sun-Yuan Wu ${ }^{1}$, Huey-Yi Chen $^{1,2}$, \\ Chiao-Hui Chang, ${ }^{1,2}$ Yuan-Ju Lee ${ }^{9}$, Tzu-Fang Hsu' ${ }^{3}$, Fuu-Jen Tsai ${ }^{1,2}$, Wei-Yong Lin ${ }^{1,2}$, Wen-Chi Chen ${ }^{1,2, *}$ \\ ${ }^{1}$ School of Chinese Medicine, Graduate Institute of Chinese Medicine, Graduate Institute of Integrated Medicine, College of Chinese \\ Medicine, Research Center for Chinese Medicine \& Acupuncture, China Medical University, Taichung, Taiwan \\ ${ }^{2}$ Departments of Dermatology, Obstetrics and Gynecology, Medical Research, and Urology, China Medical University Hospital, \\ Taichung, Taiwan \\ ${ }^{3}$ Department of Applied Cosmetology, Master Program of Cosmetic Science, HUNGKUANG University, Taiwan \\ ${ }^{4}$ Department of Psychology, College of Medical and Health Science, Asia University, Taichung, Taiwan \\ ${ }^{5}$ Center for General Education, Feng Chia University, Taichung, Taiwan \\ ${ }^{6}$ Department of Dermatology, Taichung Veteran General Hospital, Taichung, Taiwan \\ ${ }^{7}$ Department of Medicinal Botanicals and Health Applications, Da-Yeh University, Changhua, Taiwan \\ ${ }^{8}$ Department of Anesthesiology, Tungs' Taichung Harbor Hospital, Taichung, Taiwan \\ ${ }^{9}$ Department of Urology, National Taiwan University Hospital, Taipei, Taiwan \\ *Corresponding author: wgchen@mail.cmu.edu.tw
}

Received January 06, 2015; Revised February 05, 2015; Accepted February 11, 2015

\begin{abstract}
There are rising public health concerns about the links between consumption of sugar-sweetened beverages and weight gain, obesity, and other metabolic problems. Sodium citrate (Na-citrate) is used as an additive in colas and various commercial drinks worldwide. Although potassium citrate (K-citrate) has been prescribed and well accepted by urologists for treating urinary stone disease (urolithiasis), the clinical role of Na-citrate has not been well established. We investigated the effects of Na-citrate and cola on the treatment of urolithiasis with an emerging translational model - Drosophila melanogaster. Drosophila medium containing $0.5 \%$ ethylene glycol (EG) was used as a lithogenic agent for calcium oxalate (CaOx) crystal formation in Drosophila Malpighian tubules. Cola (25\%) and Na-citrate ( $2 \%$ and $4 \%$ ) were added to the fly medium for urolithiasis treatment. Medium containing Kcitrate $(2 \%$ and $4 \%$ ) was used as a positive control. After 3 weeks of treatment, the Malpighian tubules were dissected, removed, and processed for polarized light microscopy examination; fly lifespan was also monitored in different groups. Cola failed to reduce $\mathrm{CaOx}$ crystal formation, whereas Na-citrate and the positive control K-citrate significantly reduced EG-induced CaOx crystal formation in Drosophila. Administration of either Na-citrate or cola did not inhibit Drosophila lifespan. Consumption of cola exerts no detectable change in the lithogenic agent associated with $\mathrm{CaOx}$ stone formation in the Drosophila model. By contrast, Na-citrate had an inhibitory effect on EG-induced $\mathrm{CaOx}$ crystal formation, albeit a lower inhibitory rate upon comparison with K-citrate.
\end{abstract}

Keywords: cola, drosophila, sodium citrate, calcium oxalate, urolithiasis

Cite This Article: Kao-Sung Tsai, Yung-Hsiang Chen, Jui-Lung Shen, Kee-Ming Man, Sun-Yuan Wu, Huey-Yi Chen, Chiao-Hui Chang, Yuan-Ju Lee, Tzu-Fang Hsu, Fuu-Jen Tsai, Wei-Yong Lin, and Wen-Chi Chen, "Does Chronic Cola Consumption Increase Urinary Stone Risk? Evidence from the Drosophila Model of Urolithiasis.” Journal of Food and Nutrition Research, vol. 3, no. 2 (2015): 109-113. doi: 10.12691/jfnr-3-2-6.

\section{Introduction}

Soft drinks and various beverages make up an increasing percentage of energy intake, and there are rising public health concerns about the links between consumption of sugar-sweetened beverages and weight gain, obesity, and other metabolic problems [1,2,3,4,5]. Cola is an internationally popular carbonated soft drink whose contents include caffeine, phosphoric acid, sugar, and citric acid [6,7]. One major focus of concern is the potential for adverse side effects caused by consuming cola in large quantities over time $[8,9,10]$. Serial reports indicate that side effects may be attributed to caffeine, phosphoric acid, and excessive sugar intake, the latter of which may cause obesity and contribute to subsequent chronic conditions including urinary stones [11].

It is postulated that excessive long-term intake of cola may cause hypokalemia myopathy and alkalosis due to high levels of caffeine [12]. The current understanding of the effects of cola on urolithiasis is unknown, although an indirect link may exist, as urinary alkalosis may be related to stone formation $[13,14]$. By contrast, it has been 
previously reported that cola contains low levels of oxalate, which may not increase the likelihood of stone formation $[15,16]$. In one such study, the Rodgers group analyzed the urinary biochemistry of volunteers who drank 2 liters of regular cola over a period of $24 \mathrm{~h}$. Their findings indicated unfavorable changes to risk factors associated with calcium oxalate $(\mathrm{CaOx})$ stone formation [17]. However, there was insufficient clinical evidence to directly correlate long-term intake of cola beverages with stone formation. Clinical studies for urinary stone risk factors of cola intake are currently ongoing, but have yet to yield concrete results $[15,17,18,19]$. For example, Ferraro et al. showed that consuming sugar-sweetened cola had a 23\% higher risk of developing kidney stones, whereas consumption of artificially sweetened cola was associated with a trend to reduced risk [19].

Sodium citrate (Na-citrate) is a common additive of commercial cola and juice beverages. Citrate is believed to retain properties for the effective prevention of stone disease [20], and as such, potassium citrate (K-citrate) has become a common stone prevention drug in clinical settings. However, Na-citrate has been deemed less effective by some clinical studies, preventing its use in this context. A study by Sakhaee et al. found that both Nacitrate and K-citrate were effective in preventing the buildup of uric acid, however, Na-citrate was less effective [21].

A subsequent clinical study by Preminger et al. found that, although Na-citrate was able to increase urinary citrate excretion rate, it was not effective at preventing stone formation in patients with distal renal tubular acidosis [22]. Na-citrate also enhanced brushite and sodium saturation, but was not able to reduce urinary $\mathrm{CaOx}$ saturation. However, in a randomized, placebo controlled clinical trial, Allie-Hamaulay and Rodgers demonstrated that Na-citrate may alter the risk factors for $\mathrm{CaOx}$ stone [23]. Because Na-citrate is common and inexpensive, there is ample opportunity to investigate its efficacy as a preventive aid for $\mathrm{CaOx}$ stone formation.

We have developed a novel, cost-effective fruit fly model for the study of nephrolithiasis [24]. To address questions related to soft drinks, we conducted an animal study by applying our well-established fruit fly model to investigate the possible effects of cola and Na-citrate for treatment of $\mathrm{CaOx}$-related urolithiasis.

\section{Materials and Methods}

\subsection{Fly Stocks and Rearing Conditions}

We used wild type adult male flies, Drosophila melanogaster $\mathrm{CS}$, in these experiments. We used 150 to 180 flies in each group, bred in plastic vials containing standard fly medium at $25^{\circ} \mathrm{C}$ and $60 \%$ humidity with a 12 h light-dark cycle. The formula of standard fly medium consisted of $6.7 \mathrm{~g}$ agar, $21.7 \mathrm{~g}$ yeast, $13.1 \mathrm{~g}$ sugar, and $66.6 \mathrm{~g}$ corn syrup, with the addition of water to a final volume of $1 \mathrm{~L}$.

The solution was heated in the microwave, and after cooling to $85^{\circ} \mathrm{C}, 13.3 \mathrm{~mL} 99 \%$ alcohol and $3.4 \mathrm{~g} \beta$ hydroxybenzoic acid methyl ester was added. Then, 10 $\mathrm{mL}$ of medium was decanted into a $50-\mathrm{mL}$ test tube, and left to return to room temperature before its storage at $4^{\circ} \mathrm{C}$.
It should be noted that freshly prepared solution was ready for use only within a 2-week interval.

\subsection{CaOx Formation in Drosophila}

The details for the breeding of lithogenic flies are in accordance with our previous study. In brief, a solution of $0.5 \%$ ethylene glycol (EG, Sigma, St Louis, USA) was used as a lithogenic agent for $\mathrm{CaOx}$ crystal formation in Malpighian tubules. Na-citrate (2\% and 4\%) and cola (25\%) were added to the fly medium (wt/vol\%) and used as test agents, while $\mathrm{K}$-citrate medium additives (2\% and $4 \%$ ) were used as positive controls ( $n=150-180$ for each group).

The flies were subjected to $\mathrm{CO}_{2}$ narcotization, and the Malpighian tubules were dissected, removed, and processed for polarized light microscopy examination 3 weeks after breeding. Intra-tubule crystals were processed with scanning electron microscopy (SEM) and energydispersive X-ray spectroscopy (EDS) to confirm the composition as $\mathrm{CaOx}[24,25,26,27]$.

\subsection{Polarized Light Microscopy}

The Malpighian tubules were dissected and immediately observed under normal and polarized white light with an Olympus BX51 (Hicksville NY, USA) optical microscope after the $\mathrm{CaOx}$ crystal induction period. Pertinent aspects of the tubules were photographed with Kodak ProImage100 film, and scales were obtained with the projection of a micrometric slide under the same conditions used in the illustrations [24,25,26,27].

\subsection{Lifespan Assay}

Prior to conducting the lithogenic assays, we studied the effects of cola and Na-citrate on the flies' survival. To set up these lifespan assays, new emergents were collected under light $\mathrm{CO}_{2}$ anesthesia. Foam plugs were used in place of cotton ones, and food vials were kept horizontally to prevent weaker flies from accidentally adhering to food or foam.

Survivors in each vial were counted, and dead flies were removed daily. Survivorship was compared and tested for significance with log-rank tests, and lifespan curves were from pooled counts of a large number of vials $(n \cong 150)[24,25,26,27]$.

\subsection{Statistical Analyses}

Crystal formation rate was analyzed by the Chi-square test. For comparison between two lifespan curves, we determined the $P$ value in the log-rank test. All statistics were performed using the Sigma Stat software (SPSS; Systat Software, USA).

\section{Results}

\subsection{CaOx crystal Formation}

EG-induced crystal formation in Drosophila Malpighian tubules was clearly observed using microscopy (Figure 1) and was previously identified as $\mathrm{CaOx}$. 

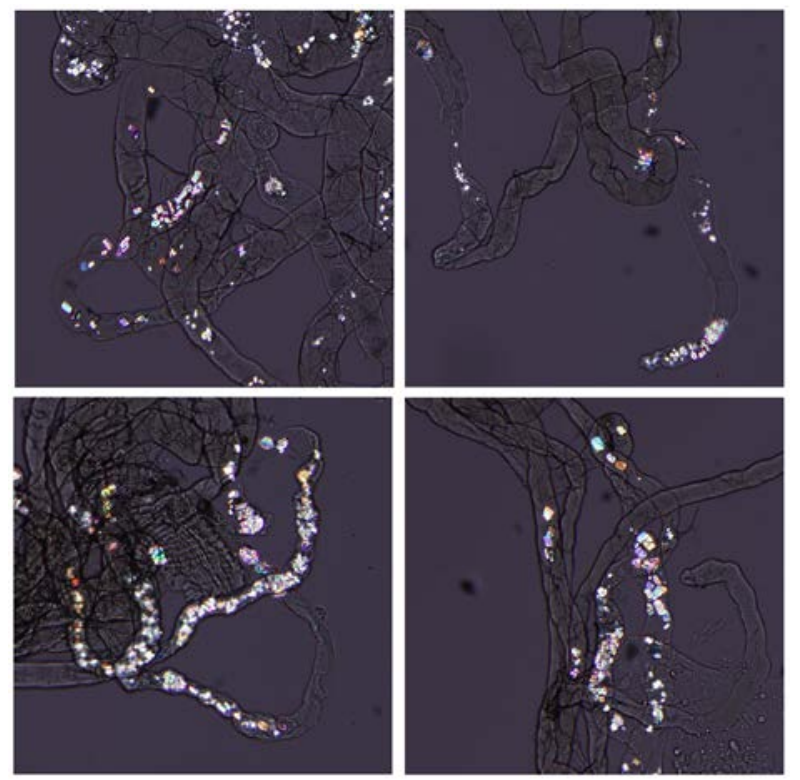

Figure 1. EG-induced CaOx crystal deposition in the Malpighian tubules of Drosophila. The images show representative polarized microscopy for the flies with 0.5\% EG-induced crystal formation in Malpighian tubules

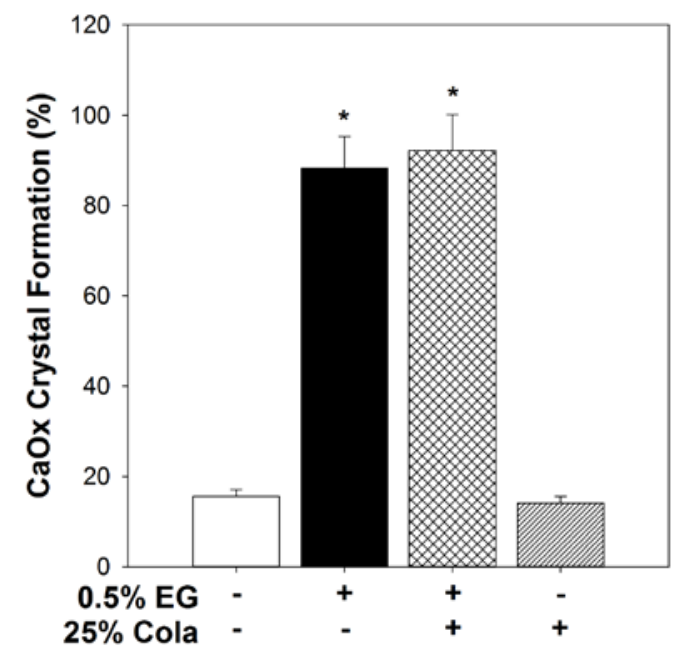

Figure 2. Crystal formation in $0.5 \%$ EG and/or $25 \%$ cola treated Drosophila ( $\mathrm{n} \cong 150$ for each group). $* P<0.05$, compared to the control

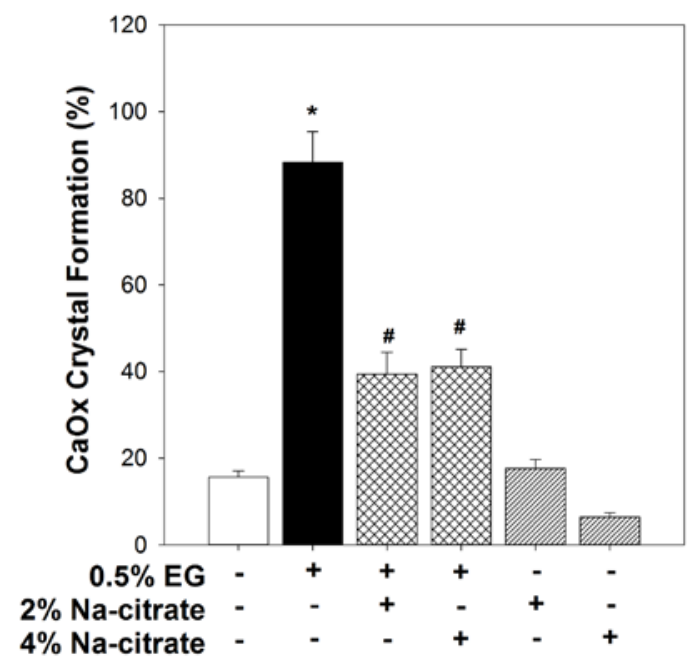

Figure 3. Crystal formation in $0.5 \%$ EG and/or Na-citrate ( $2 \%$ and $4 \%$ ) treated Drosophila ( $\mathrm{n} \cong 150$ for each group). ${ }^{*} P<0.05$, compared to the control. ${ }^{\#} P<0.05$, compared to the $0.5 \%$ EG-treated group
The rates of $\mathrm{CaOx}$ crystal formation in the control group and $0.5 \%$ EG group were $15.6 \%$ and $88.3 \%$, respectively. The rate of crystal formation in the group of EG+cola was $92.2 \%$ and was not different from the lithogenic group $(P=0.245$; Figure 2$)$. Single added agents such as cola (Figure 2) and Na-citrate (Figure 3) without EG did not increase the rate of crystal formation. Both K-citrate (Figure 3) and Na-citrate (Figure 4) revealed significant inhibitory effects on crystal formation $(P=0.026)$.

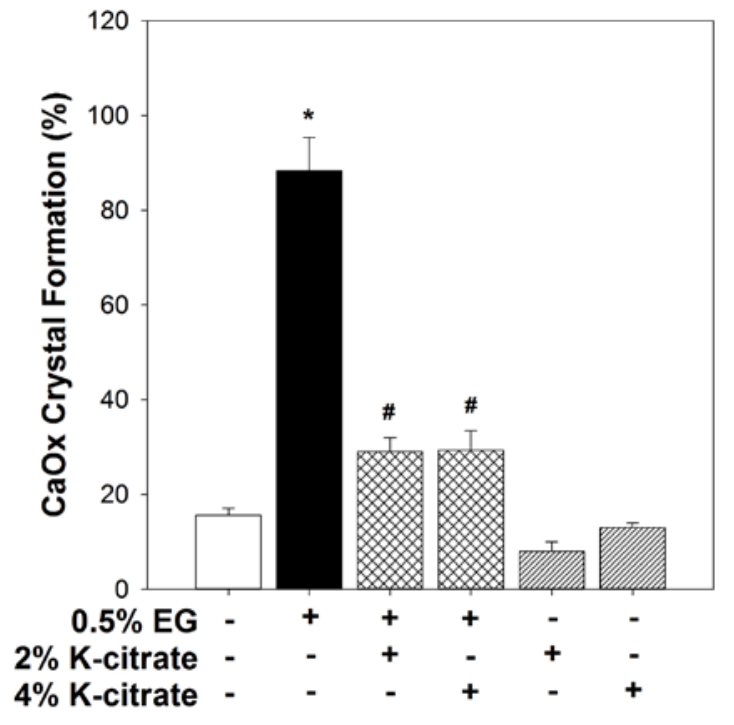

Figure 4. Crystal formation in $0.5 \% \mathrm{EG}$ and/or K-citrate ( $2 \%$ and $4 \%$ ) treated Drosophila ( $\cong 150$ for each group). ${ }^{*} P<0.05$, compared to the control. ${ }^{\#} P<0.05$, compared to the $0.5 \%$ EG-treated group

\subsection{Drosophila LIFESPAN}

To test whether the effects of cola or Na-citrate dosage were associated with an increased mortality rate, the lifespans of Drosophila were measured after supplementing their food with cola or Na-citrate. The mean life span was not significantly reduced by administration of both agents, and K-citrate comparing with control group (Figure 5).

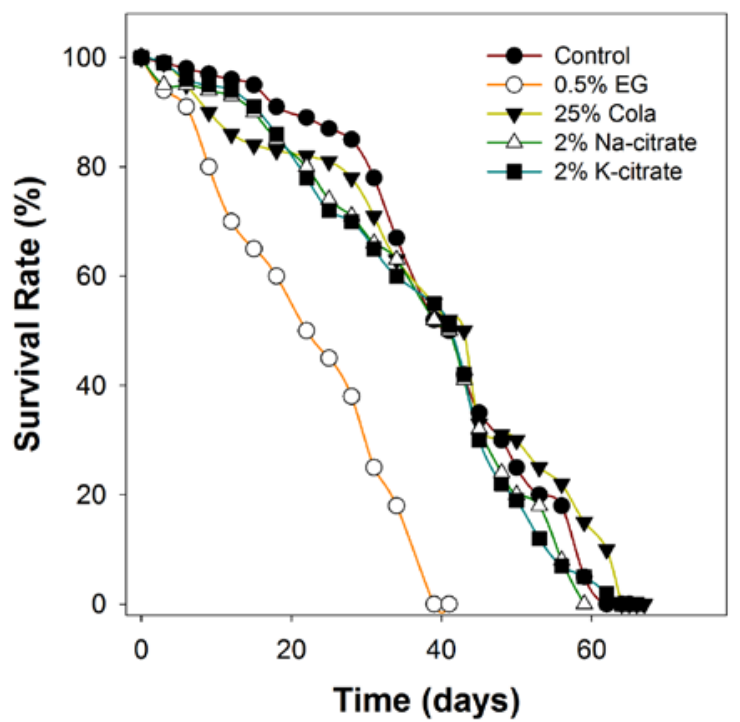

Figure 5. Lifespan of $0.5 \%$ EG, 25\% cola, 2\% Na-citrate, and 2\% Kcitrate-treated flies 


\section{Discussion}

According to the emerging translational Drosophila model of urolithiasis, cola did not promote or inhibit $\mathrm{CaOx}$ crystal formation. Administration of $2 \% \mathrm{Na}$-citrate exerted an inhibitory effect estimated at $39.4 \%$ on EGinduced $\mathrm{CaOx}$ crystal formation, similar to that of $\mathrm{K}$ citrate administration (29.0\%).

Cola is a common commercial drink that is distributed worldwide [28]. Na-citrate has long been used as a drink additive, yet there is concern about its side effects in a long-term beverage. In this study, there was no difference in the lifespan of Drosophila treated with Na-citrate or cola compared with control treatments. Moreover, the addition of cola neither prevented nor enhanced the effects $\mathrm{CaOx}$ crystal formation in the Malpighian tubules. Cola contains glucose/fructose, caffeine, water and some confidential components [29]. Long-term consumption of high sugar beverages may contribute to many diseases such as insulin resistance, obesity, hypertensive cardiovascular disease, and kidney disease [30]. However, there was no reported association of the cola with patients' health [31,32]. Caffeine is one of the major contents of cola that has previously been reported responsible for the formation of $\mathrm{CaOx}$ stones [12]. Interestingly, Curhan et al. conducted a 6-year follow-up study by means of a semi-quantitative food frequency questionnaire that found that those who drink a caffeine-containing beverage daily lowered their risk of stone recurrence by $10 \%$ [33]. Furthermore, Herrel et al. studied urinary stone risk factors in 13 participants (3 controls) with cola consumption and found no detectable change. They concluded that cola consumption did not contribute to the risk of developing urinary stone disease [15]. Therefore, it is possible that in a clinical context, cola may not exhibit the lithogenic effect that we have shown with our Drosophila data.

The clinical, therapeutic effect of K-citrate in preventing $\mathrm{CaOx}$ crystal formation has been well documented and confirmed [34]. Gao et al. reported that K-citrate significantly increased the successful expulsion rate of melamine-induced urinary calculi [35]. K-citrate has been used clinically for its inhibitory effects on the accumulation of calcium and uric acid stones [36], and because these effects are due to citrate regardless of sodium or potassium. Our results showed Na-citrate having an inhibitory effect similar to that of K-citrate, which argues the potential for its future use in a clinical context. In a study of the alkali action on urinary crystallization of calcium salts by Preminger et al., Kcitrate, but not Na-citrate, had an effect on the retardation of CaOx saturation in urine. Thereafter, K-citrate has been used clinically as a preventive drug for $\mathrm{CaOx}$ stone recurrence, which has been widely reported [22]. Owing to its common use in drinks as an additive, we wonder whether Na-citrate has any contrasting effects; our results showed a therapeutic effect. Therefore, further study of the clinical effects of Na-citrate is warranted to clarify its role in the prevention of $\mathrm{CaOx}$ stone recurrence.

Our study has several advantages, yet is not without limitations. We have applied a novel animal model [37], which readily provided a large number of test subjects. Moreover, the experimental period was short and the crystal components in the Malpighian tubules were easily detected through SEM with EDS. In addition, the results of our study were consistent with data from rats and humans [38]. However, the weakness of non-mammalian Drosophila model is still a limitation of our study; flies are invertebrate animals, and as such, our model may not be fully indicative of the data we would obtain from mammals. Further studies are warranted to confirm the insect data, especially considering that we provided cola in the fly food daily; this may have exerted additive effects that may not be translatable to human drink habits, unless cola were to be consumed daily.

\section{Conclusions}

Chronic cola load alone does not affect crystal formation. Na-citrate and K-citrate have a similar inhibitory effect on $\mathrm{CaOx}$ crystal formation in our fly model. The ability to readily manipulate and quantify stone formation in these Drosophila models presents a unique opportunity to increase our understanding of urolithiasis.

\section{Acknowledgement}

The authors declare that they have no conflict of interest. This study is supported in part by Taiwan Ministry of Health and Welfare Clinical Trial and Research Center of Excellence (MOHW104-TDU-B-212113002), Taiwan Ministry of Science and Technology (NSC102-2314-B-039-025), China Medical University (CMU103-S-19), and CMU under the Aim for Top University Plan of the Taiwan Ministry of Education (A5-2-A). K.S.T. and Y.H.C. contributed equally to this study.

\section{References}

[1] V. Andres, M. J. Villanueva, I. Mateos-Aparicio, and M. D. Tenorio, "Colour, bioactive compounds and antioxidant capacity of mixed beverages based on fruit juices with milk or soya," Journal of Food and Nutrition Research, 53 (1). 71-80. 2014.

[2] M. Kockova, J. Mendel, A. Medvedova, E. Sturdik, and L. Valik, "Cereals and pseudocereals as substrates for growth and metabolism of a probiotic strain Lactobacillus rhamnosus GG," Journal of Food and Nutrition Research, 52 (1). 25-36. 2013.

[3] Z. Kohajdova, J. Karovicova, and M. Greifova, "Analytical and organoleptic profiles of lactic acid-fermented cucumber juice with addition of onion juice," Journal of Food and Nutrition Research, 46 (3). 105-111. 2007.

[4] A. Steskova, M. Morochovicova, and E. Leskova, "Vitamin C degradation during storage of fortified foods," Journal of Food and Nutrition Research, 45 (2). 55-61. 2006.

[5] M. Tuzen, S. Saracoglu, and M. Soylak, "Evaluation of trace element contents of powdered beverages from Turkey," Journal of Food and Nutrition Research, 47 (3). 120-124. 2008.

[6] J. I. Friedlander, J. A. Antonelli, and M. S. Pearle, "Diet: from food to stone," World Journal of Urology. 2014.

[7] S. D. Ladas, D. Kamberoglou, G. Karamanolis, J. Vlachogiannakos, and I. Zouboulis-Vafiadis, "Systematic review: Coca-Cola can effectively dissolve gastric phytobezoars as a firstline treatment," Alimentary Pharmacology and Therapeutics, 37 (2). 169-173. 2013.

[8] C. H. Wang, W. D. Lin, D. T. Bau, and I. C. Chou, "Appearance of acanthosis nigricans may precede obesity: An involvement of the insulin/IGF receptor signaling pathway," BioMedicine, 3 (2). 82-87. 2013.

[9] P. L. Wu, H. Y. Lane, H. S. Tang, and G. E. Tsai, "Glutamate theory in developing novel pharmacotherapies for obsessive 
compulsive disorder: Focusing on N-methyl-D-aspartate signaling," BioMedicine, 2 (2). 75-79. 2012.

[10] C. C. Lee, C. H. Tsai, L. Wan, and Y. Tsai, "Increased incidence of Parkinsonism among Chinese with $\beta$-glucosidase mutation in central Taiwan," BioMedicine, 3 (2). 92-94. 2013.

[11] S. Nowfar, K. Palazzi-Churas, D. C. Chang, and R. L. Sur, "The relationship of obesity and gender prevalence changes in United States inpatient nephrolithiasis," Urology, 78 (5). 1029-1033. 2011.

[12] J. E. Rice, and J. D. Faunt, "Excessive cola consumption as a cause of hypokalaemic myopathy," Internal Medicine Journal, 31 (5). 317-318. 2001.

[13] L. Shen, X. Sun, H. Zhu, X. Cong, and B. Ning, "Comparison of renal function and metabolic abnormalities of cystine stone patients and calcium oxalate stone patients in China," World Journal of Urology, 31 (5). 1219-1223. 2013.

[14] O. Karagulle, U. Smorag, F. Candir, et al., "Clinical study on the effect of mineral waters containing bicarbonate on the risk of urinary stone formation in patients with multiple episodes of CaOx-urolithiasis," World Journal of Urology, 25 (3). 315-323. 2007.

[15] L. Herrel, J. Pattaras, T. Solomon, and K. Ogan, "Urinary stone risk and cola consumption," Urology, 80 (5). 990-994. 2012.

[16] G. P. Kasidas, and G. A. Rose, "Oxalate content of some common foods: determination by an enzymatic method," Journal of Human Nutrition, 34 (4). 255-266. 1980.

[17] A. Rodgers, "Effect of cola consumption on urinary biochemical and physicochemical risk factors associated with calcium oxalate urolithiasis," Urological Research, 27 (1). 77-81. 1999.

[18] C. M. Passman, R. P. Holmes, J. Knight, et al., "Effect of soda consumption on urinary stone risk parameters," Journal of Endourology, 23 (3). 347-350. 2009.

[19] P. M. Ferraro, E. N. Taylor, G. Gambaro, and G. C. Curhan, "Soda and other beverages and the risk of kidney stones," Clinical Journal of the American Society of Nephrology, 8 (8). 1389-1395. 2013.

[20] T. Esen, A. Krautschick, and P. Alken, "Treatment update on pediatric urolithiasis," World Journal of Urology, 15 (3). 195-202. 1997.

[21] K. Sakhaee, M. Nicar, K. Hill, and C. Y. Pak, "Contrasting effects of potassium citrate and sodium citrate therapies on urinary chemistries and crystallization of stone-forming salts," Kidney International, 24 (3). 348-352. 1983.

[22] G. M. Preminger, K. Sakhaee, and C. Y. Pak, "Alkali action on the urinary crystallization of calcium salts: contrasting responses to sodium citrate and potassium citrate," Journal of Urology, 139 (2). 240-242. 1988

[23] S. Allie-Hamdulay, and A. L. Rodgers, "Prophylactic and therapeutic properties of a sodium citrate preparation in the management of calcium oxalate urolithiasis: randomized, placebocontrolled trial," Urological Research, 33 (2). 116-124. 2005.
[24] Y. H. Chen, H. P. Liu, H. Y. Chen, et al., "Ethylene glycol induces calcium oxalate crystal deposition in Malpighian tubules: a Drosophila model for nephrolithiasis/urolithiasis," Kidney International, 80 (4). 369-377. 2011.

[25] W. C. Chen, W. Y. Lin, H. Y. Chen, et al., "Melamine-induced urolithiasis in a Drosophila model," Journal of Agricultural and Food Chemistry, 60 (10). 2753-2757. 2012.

[26] C. Y. Ho, Y. H. Chen, P. Y. Wu, et al., "Effects of commercial citrate-containing juices on urolithiasis in a Drosophila model," Kaohsiung Journal of Medical Sciences, 29 (9). 488-493. 2013.

[27] S. Y. Wu, J. L. Shen, K. M. Man, et al., "An emerging translational model to screen potential medicinal plants for nephrolithiasis, an independent risk factor for chronic kidney disease," Evidence-Based Complementary and Alternative Medicine, 2014. 972958. 2014.

[28] T. H. de Sa, "Can Coca Cola promote physical activity?," Lancet, 383 (9934). 2041. 2014.

[29] P. Maes, Y. B. Monakhova, T. Kuballa, H. Reusch, and D. W. Lachenmeier, "Qualitative and quantitative control of carbonated cola beverages using (1)H NMR spectroscopy," Journal of Agricultural and Food Chemistry, 60 (11). 2778-2784. 2012.

[30] S. Wang, J. Chen, and M. Valderrabano, "Nutrient restriction preserves calcium cycling and mitochondrial function in cardiac myocytes during ischemia and reperfusion," Cell Calcium, 51 (6). 445-451. 2012.

[31] M. Aoyagi, and K. Nagata, "Learning coefficient of generalization error in Bayesian estimation and vandermonde matrix-type singularity," Neural Computation, 24 (6). 1569-1610. 2012.

[32] P. Celec, "Intake of cola beverages containing caffeine does not increase, but reduces body weight," European Journal of Clinical Nutrition, 66 (4). 538; author reply 539. 2012.

[33] G. C. Curhan, W. C. Willett, E. B. Rimm, D. Spiegelman, and M. J. Stampfer, "Prospective study of beverage use and the risk of kidney stones," American Journal of Epidemiology, 143 (3). 240247. 1996.

[34] Y. H. Lee, W. C. Huang, J. Y. Tsai, and J. K. Huang, "The efficacy of potassium citrate based medical prophylaxis for preventing upper urinary tract calculi: a midterm followup study," Journal of Urology, 161 (5). 1453-1457. 1999.

[35] J. Gao, Y. Shen, N. Sun, et al., "Therapeutic effects of potassium sodium hydrogen citrate on melamine-induced urinary calculi in China," Chinese Medical Journal (Engl), 123 (9). 1112-1116. 2010.

[36] I. P. Heilberg, and D. S. Goldfarb, "Optimum nutrition for kidney stone disease," Advances in Chronic Kidney Disease, 20 (2). 165174. 2013.

[37] F. Knauf, and P. A. Preisig, "Drosophila: a fruitful model for calcium oxalate nephrolithiasis?," Kidney International, 80 (4). 327-329. 2011.

[38] W. L. Liao, and F. J. Tsai, "Personalized medicine: A paradigm shift in healthcare," BioMedicine, 3 (2). 66-72. 2013. 